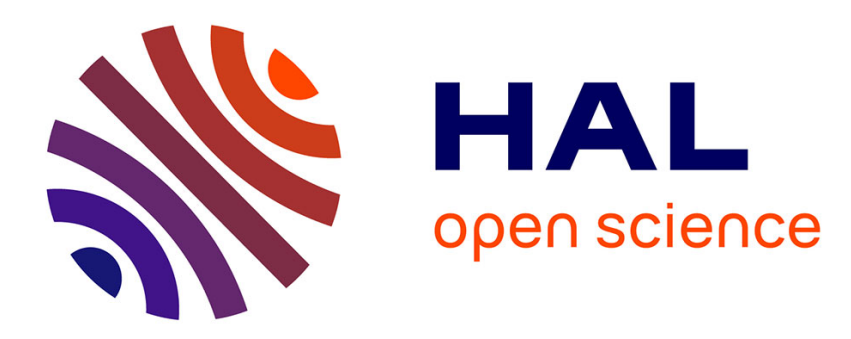

\title{
Neurohistiocytose : de la classification clinique à la thérapie ciblée
}

\author{
Ahmed Idbaih
}

\section{To cite this version:}

Ahmed Idbaih. Neurohistiocytose: de la classification clinique à la thérapie ciblée. Revue Neurologique, 2015, 171 (supplément 1), pp.A211. 10.1016/j.neurol.2015.01.480 . hal-01132901

\section{HAL Id: hal-01132901 https://hal.sorbonne-universite.fr/hal-01132901}

Submitted on 18 Mar 2015

HAL is a multi-disciplinary open access archive for the deposit and dissemination of scientific research documents, whether they are published or not. The documents may come from teaching and research institutions in France or abroad, or from public or private research centers.
L'archive ouverte pluridisciplinaire HAL, est destinée au dépôt et à la diffusion de documents scientifiques de niveau recherche, publiés ou non, émanant des établissements d'enseignement et de recherche français ou étrangers, des laboratoires publics ou privés. 


\title{
Neurohistiocytose : de la classification clinique à la thérapie ciblée
}

\author{
Ahmed Idbaih \\ AP-HP, Groupe Hospitalier Pitié-Salpêtrière, Service de neurologie 2-Mazarin, Paris, France. \\ Sorbonne Universités, UPMC Univ Paris 06, UM 75, ICM, F-75013 Paris, France \\ Inserm, U 1127, ICM, Paris, F-75013 Paris, France \\ CNRS, UMR 7225, ICM, Paris, F-75013 Paris, France \\ ICM, Paris, 75013 France
}

Les histiocytoses regroupent des maladies multisystémiques hétérogènes caractérisées par la prolifération de cellules du lignage monocytes-macrophages-cellules dendritiques. Trois grands groupes d'histiocytoses, eux-mêmes subdividés en plus entités, sont individualisés: (i) dendritiques, (ii) macrophagiques, et (iii) malignes.

Les histiocytoses dendritiques sont dominées par l'histiocytose langerhansienne, autrefois appelée histiocytose « $\mathrm{X}$ » résultant de la fusion de plusieurs entités (i.e. le granulome éosinophile, la maladie de Hand-Schüller-Christian, et la maladie de Abt-Letterer-Siwe). Les histiocytoses dendritiques non langerhansiennes regroupent notamment le xanthogranulome juvénile et la maladie d'Erdheim-Chester. La lymphohistiocytose hémophagocytique primitive et la maladie de Rosai-Dorfman font, quant à elles, partie des histiocytoses macrophagiques. Enfin, les histiocytoses malignes regroupent certaines leucémies et certains sarcomes.

Les histiocytoses touchent le système nerveux central avec deux principaux types de lésions: (i) pseudotumorale et (ii) pseudodégénérative. Ces lésions s'opposent en tous points. En effet, l'épidémiologie, les manifestations cliniques, l'aspect neuroradiologique, les caractéristiques histologiques et le pronostic de ces types de lésions observées dans les histiocytoses sont radicalement différents.

Ces dernières années ont été marquées par la découverte de mutations activatrices récurrentes du gène $B R A F$ dans les histiocytoses. Des thérapies moléculaires ciblées contre le produit de ces anomalies génétiques ont été développées. Elles ont révolutionné le pronostic des lésions pseudotumorales systémiques et neurologiques des histiocytoses avec des régressions lésionnelles importantes.

La classification diagnostique des histiocytoses et de leurs atteintes neurologiques ont été raffinées grâce notamment à la microscopie électronique, à la biologie moléculaire et à l'imagerie. Cette meilleure caractérisation multimodale des histiocytoses a également ouvert la voie à des stratégies thérapeutiques innovantes. 